\title{
The Ethics of Developing New Treatments: A Case Study of the West African Ebola Outbreak and the Use of Randomized Control Trials

\author{
Nelly Noubossi*, Biology
}

\begin{abstract}
The 2014-2015 Ebola epidemic was the most devastating Ebola outbreak in history, killing over 10,000 people. During the outbreak, the WHO led efforts to design the best method to test the potential treatments quickly. Randomized controlled trials (RCTs) were proposed as the best method, although many experts opposed their use, deeming them inappropriate in the context of an epidemic. Despite the long debate, RCTs were used to test the available treatments. This paper presents arguments both supporting and opposing RCTs, and analyzes a few example RCTs conducted to answer the following question: "were RCTs effective at helping researchers fight the epidemic?" This paper argues that RCTs were not the best approach for two reasons: the principle of equipoise requires that patients are provided available treatments; if RCTs were to be used, they should have begun earlier to ensure the validity of the findings.
\end{abstract}

\section{INTRODUCTION:}

Ebola virus disease (EVD) is a severe and often fatal illness in humans. EVD first appeared in 1976 when two outbreaks occurred simultaneously in present-day South Sudan and Democratic Republic of Congo ("Ebola virus disease," 2018). There have been five identified strains of the Ebola virus, but three of them (Zaire, Sudan, Bundibugyo) are responsible for the majority of cases in humans (Coltart, Lindsey, Ghinai, Johnson, \& Heymann, 2017). The Ebola virus is introduced into human populations through contact with the blood, secretions, and bodily fluids of infected animals such as fruit bats and chimpanzees ("Ebola virus disease," 2018). From there, it spreads between humans through the same mechanism . On March 23, 2014, the WHO's regional office in Guinea reported an outbreak of EVD ("Ebola virus disease," 2018). This outbreak spread to nearby counties and primarily affected three West African countries: Guinea, Sierra Leone and Liberia. Nigeria was also infected.. It was the most devastating Ebola outbreak

\footnotetext{
* Nelly Nouboussi is a third-year biology major, with minors in Chemistry and Global health. Originally from Cameroon, central Africa, she moved to the United stated when she was 13 to continue her education. She plans to graduate from college and attend medical school to become a neurosurgeon. She volunteers at at the Riverbend Hospital, is the co-director of the African Student Association and conducts research in a research lab at the Institute of Neuroscience on campus, which led to her being able to present her research at the University's annual undergraduate symposium. She has participated in a global health-related internship in Accra, Ghana during which she learned more about the healthcare system in the country. She is also a pathway mentor for 1 st-year students and a biology tutor. Her hobbies include running, watching tv and performing African dance. Her long-term goals include returning to Cameroon to contribute to the development of a better healthcare system. Please direct correspondence to nnoubous@uoregon.edu.
} 
in history as 28,646 cases and 11,223 deaths were reported, with the mortality rate in some regions as high as 70\% (Coltart et al., 2017).

During the outbreak, efforts led by the WHO focused on designing the best method to gather data about available treatments quickly. Drugs such as Zmapp and vaccines such as ChAd3 demonstrated effectiveness against Ebola in primates, but they had not yet been tested in humans. The gold standard for clinical trials-double-blind Randomized Controlled Trials (RCTs)-was proposed, but there were ethical questions regarding its appropriateness in the context of an epidemic. The first part of this paper will outline the key arguments given by proponents and opponents of RCTs and will also discuss some proposed alternative study designs. The second part of the paper will analyze five of the RCTs conducted to assess their safety and efficacy. While there were significant arguments for and against RCTs, I argue they were not the best approach during the Ebola outbreak because the ethical principle of equipoise required distributing the treatments to all patients in this situation, not just those in the "treatment" group of a RCT. If they were to be conducted, RCTs should have started earlier to ensure a large enough sample size for the data to be valid and useful.

\section{ARGUMENTS FOR RANDOMIZED CONTROLLED TRIALS}

Multiple arguments were proposed to justify the need to conduct RCTs, the main one being that they ensure the validity of data. In principle, RCTs balance out all the confounding variables and ensure comparability between treatment and control groups (Upshur \& Fuller 2016). The goal is to decrease the likelihood of any observed effect being the result of variables other than the treatment. Lanini et al. (2015) focus on the placebo control group to justify the use of RCTs. The authors argue that having a placebo control allows for clear contrast between the two groups, making even minor differences detectable.. In other words, since the only difference expected between the two groups is the experimental drug, even small differences would be attributed to the drug. Otherwise, the lack of a placebo control group would create uncertainty as to whether the effect is due to the experimental treatment or some other factor. Furthermore, the authors argue that being able to generate reliable data quickly using RCTs is important because researchers will be armed for future outbreaks . Because uncertainty might result from using experimental treatments in non-randomized studies, researchers will not be sure that these drugs will be effective again in the future. Therefore, RCTs should be conducted to test experimental treatments because they provide the most valid results and thus prepare the global community for subsequent outbreaks.

Another reason why researchers believe RCTs should be conducted is that they ensure the safety of the patients. The main ethical principle behind this argument is equipoise, which means that there is genuine uncertainty among clinical experts over the risks or benefits of an untested clinical treatment (Adebamowo et al., 2014). In other words, if healthcare workers are truly uncertain about the potential risks associated with the treatment, randomization is the safest avenue. Clifford Lane, deputy director for clinical research and special projects at the US National Institute of Allergy and Infectious Diseases put it best: "the idea that there is no need for controlled trials presupposes that the drugs have zero side effects, that they are efficacious, and 
that there's no substantial variability from patient to patients [...] I don't think any of that is true" (Hayden, 2014). Moreover, Philippe Calain, a researcher for MSF (Doctors Without Borders), states that the efficacy of a treatment on animals is not always a good predictor of its effect on humans (Calain, 2016). He says this in response to the proposition that one of the experimental vaccines shown to be effective in chimpanzees be distributed to all Ebola patients without conducting RCTs ("Ethical Considerations for Use of Unregistered Interventions for Ebola Virus Disease (EVD)," 2014). This proposition was made during a panel discussion organized by the WHO in August 2014. Hence, the potential that the drug might show adverse effects to humans as opposed to non-human primates explains the need to conduct RCTs first.

Additional arguments in favor of RCTs focus on the scarcity of resources in affected countries. The first argument is that limited funding and resources demand that treatments be used to generate reliable data quickly -any other use would be wasteful. According to Lanini et al. (2015), even a small quantity of treatments can be used in RCTs to generate significant data about their effectiveness; using them in non-randomized trial would reduce their potential and be wasteful . The authors argue that since not everyone can be helped, it makes the most sense to use the few treatments available to acquire knowledge which will be more impactful for the present and future. In "Evaluating Ebola Therapies - The case for RCTs," the authors take it one step further by arguing that since the treatments are limited in quantity, RCTs are not actually depriving anyone from the drugs and should instead be used to generate reliable data (Cox, Borio, \& Temple, 2014). The authors argue that the patients in these West African countries would not have had access to the treatment anyways, and thus they are not being put in more danger by participating in an RCT or being excluded from one.

Additionally, the limited resources demand that patients be altruistic and accept that the burdens and benefits of research will not be distributed equally. Lanini et al. (2015) ask the difficult questions very clearly: "How do you distribute treatments that are not produced on a mass-scale in non-randomized studies? Who gets it and who doesn't?". The authors make the point that even if we wanted to distribute the treatments widely, it is not feasible. This is mainly because of the high cost of treatments and the lack of health infrastructure in affected countries. Philippe Calain (2016) attempts to provide an answer by arguing that patients should choose to enroll in these trials for the greater good. In other words, patients should accept the risk of being placed in a placebo group and forget about their self-interests. There is no need to answer the question above, as patients would choose to give up the potential benefits they may obtain from an experimental drug for the greater good.

Calain's argument assumes that all patients are altruistic and, even in an epidemic, will act in the best interest of society. His argument also outlines the main goal of Randomized Controlled Trials (RCTs): to generate robust data that contribute to the advancement of knowledge and ultimately benefit future patients (Calain, 2016). The inherent goal of RCTs is not to benefit individual patients, but to benefit society as a whole, and more specifically future generations. This also suggests that the controversy over the use of RCTs stems from the misunderstanding of the goal of these trials by those who oppose their use. Therefore, scarcity of resources is used as 
the primary reason to explain that the priority should be to generate data quickly through RCTs, and that patients should choose to enroll in these trials for the sake of the greater good.

\section{ARGUMENTS AGAINST RANDOMIZED CONTROLLED TRIALS}

Multiple arguments have also been put forward to explain why RCTs are not ethical. RCTs, as argued by some health care workers, cause mistrust between patients and their healthcare system. The article "Randomized controlled trials: practical and ethical issues," was written by authors from various countries, including those affected by the Ebola outbreak (Guinea, Nigeria, Liberia). In this article, the authors discuss issues that healthcare workers in these countries face. They state that because patients are already terrified due to the epidemic and already lack trust in healthcare workers, they will be even more distrustful if they know that potential treatments are withheld for the purpose of research (Adebamowo et al. 2014). The authors believe that the patients would not understand the rationale behind them receiving the "standard of care" (nothing or a placebo) when other options are available. Additionally, doctors' therapeutic obligation to treat their patients i conflicts directly with randomization (Calain, 2016). If doctors have to enroll their patients into RCTs and some are placed in the placebo group, the doctorpatient relationship will be compromised because patients cannot be sure that doctors are acting in their best interest. Thus, the preservation of patients' trust and doctors' obligation to treat justifies the need to avoid RCTs.

The context of a disease epidemic is also given as a reason for why RCTs are inappropriate, since they are neither ethically acceptable nor feasible. According to Adebamowo et al. (2014), the concept of equipoise, which was discussed earlier as justification for RCTs, breaks down "when conventional care offers little benefit and mortality is extremely high." The Ebola outbreak was rapidly claiming people's lives, with mortality rates as high as 70\% in some regions (Adebamowo et al. 2014). In other words, because conventional care was ineffective, any experimental treatment would most likely yield more benefits than harm and should therefore be used. Jeremy Farrar, head of the Wellcome Trust and an infectious disease researcher, argues that RCTs are just as problematic for sick healthcare workers when he asks: "if you were there tomorrow and you were a healthcare worker, would you be willing to be in a control arm when the next three months you will be looking after patients with Ebola?" (Cohen \& Kupferschmidt, 2014). This implies that local healthcare workers would be just as likely to take the risk. Hence, RCTs are not ethically acceptable to both patients and workers because high mortality rates minimize the risk of taking an experimental treatment.

Additionally, RCTs are not feasible in the context of an epidemic. First of all, when patients are terrified due to an Ebola outbreak, they cannot offer truly voluntary informed consent (Adebamowo et al. 2014). This means that patients would be too distracted by their deteriorating health and the conditions around them to make an informed decision regarding the trials. Moreover, it might not be possible to control conditions during an epidemic because the trials would be conducted in areas where healthcare systems are breaking down and fear has been instilled in the communities (Adebamowo et al., 2014; Hayden, 2014; Kanapathipillai et al., 2014). This argument is significant since one of the reasons why RCTs are considered superior is because 
they balance confounding variables and ensure that the only difference between the two groups is the experimental treatments. However, if the trials are conducted in settings were healthcare systems are broken and people are scared, anxious and distrustful of health facilities, it may not be possible to control for all the factors that could affect the results.

The final argument against RCTs directly attacks the claim that they produce more reliable and valid data. In their article "Randomized Controlled Trials in the West African Ebola virus outbreak," Ross Upshur and Jonathan Fuller (2016) argue that it is impossible to balance out all the confounding variables. They state that "all studies fall short of the guarantee of pristine group comparability and that this ideal is not required". They believe that the absence of selection bias is necessary when a study is only looking for a small outcome, whereas investigators who are designing Ebola treatments want to see large treatment effects. They argue that if a drug is effective, it should have a drastic effect on patients' survival rate, which would be noticeable even in non-randomized studies, rendering RCTs unnecessary. This is in contrast to Lanini et al. (2015) article which argues that RCTs are necessary because they will enable researchers to detect small effects. The difference in opinion comes from whether researchers are looking for small or large outcomes; Upshur and Fuller would argue that large outcomes are needed when the mortality rate is so high. They end their article by stating that there is no gold standard, as the best design depends on the purpose and context, which is also a point made in other articles (Adebamowo et al. 2014; Hayden 2014; Upshur and Fuller 2016).

\section{ALTERNATIVE DESIGNS}

Most of the articles that argue against RCTs propose alternative approaches, two of which will be discussed. The most emphasized design was the adaptive RCT. In this type of study, the trial is modified as data is generated (Calain, 2016; Lanini et al., 2015). For example, if a large number of people die in the control group compared to the experimental group during the first few weeks of the trial, the trial would be modified so that more people in the control group would receive the drug. In other words, the likelihood that the trial will end depends on the mortality rate in the control group (Adebamowo et al. 2014). This would minimize the number of people in the control group who do not receive the treatment. The reasoning behind this design is that if a treatment is extraordinarily effective, it would be evident early on and the trial would end early so that everyone can receive the new treatment (Lanini et al. 2015). Alternatively, if the drug is not very effective, the difference between the two groups will probably not be significant, and the trial would continue as planned. An adaptive design preserves the scientific superiority of RCTs while ensuring that most people receive the treatment on time.

Another alternative is the stepped wedge design, which ensures that all people receive vaccines, but at different time periods. In this design, as shown in figure 1, participants are randomly assigned to different groups which receive the vaccine at one of several time periods (Kanapathipillai et al. 2014). Outcomes in each group are measured at each point where the next group receives the vaccine (Kanapathipillai et al. 2014). This allows researchers to gather data sequentially and to compare the results of groups at different time points. According to virologist Barney Graham, such a design would be more ethically acceptable to patients because everyone 
eventually receives the treatment (Cohen \& Kupferschmidt, 2014). Therefore, similar to the adaptive design, such a technique would decrease the likelihood that a large number of people do not receive the treatment and is thus more ethically acceptable than traditional RCTs.

\begin{tabular}{cccccc}
\multicolumn{7}{c}{ Time of Intervention } \\
Group & T1 & T2 & T3 & T4 & T5 \\
\hline A & 0 & X & & & \\
\hline B & 0 & 0 & $X$ & & \\
\hline C & 0 & 0 & 0 & $X$ & \\
\hline D & 0 & 0 & 0 & 0 & $X$ \\
\hline
\end{tabular}

Figure 1. Stepped-wedge study design (Kanapathipillai et al. 2014)

\section{WAS THE APPROACH EFFECTIVE?}

Despite all the proposed arguments against RCTs, multiple RCTs were conducted in West Africa and abroad for the candidate drugs and vaccines that were available in 2014-2015. Three of these treatments will be discussed: the triple monoclonal antibody cocktail Zmapp, the chimpanzee adenovirus type-3 vector-based Ebola Zaire vaccine (ChAd3) and the recombinant vesicular stomatitis virus vaccine (rVSV). Five randomized studies conducted between 2014 and 2015 will be analyzed to argue that RCTs were not the best method to effectively address the 20142015 epidemic because the principle of equipoise requires distributing the drugs to all patients, not just those enrolled in the treatment arm. Alternatively, if they were to be conducted, RCTs should have been started earlier to ensure a large enough sample size for the data to be valid and useful.

The first reason why RCTs were not the best approach is that these studies all showed that the experimental treatments were safe, immunogenic and/or partially effective. A study conducted between September and November 2014 tested the safety and immunogenicity of ChAd3 on 60 adult volunteers in Oxford, United Kingdom (Ewer et al., 2016). The participants received one injection of the vaccine at three different doses. The investigators did not identify any safety concerns and determined that the vaccine was immunogenic (may help the body mount an immune response against the Ebola virus) at the three different doses (Ewer et al., 2016). Another study conducted between October 2014 and February 2015 tested the safety and immunogenicity of ChAd3 on 91 participants in Mali and 20 in the US (Tapia et al., 2016). The participants received either different doses of the vaccine or a placebo. Again, the vaccine was safe and the investigators determined the dose of the vaccine that could be used in ring-vaccinations to provide "high-level 
protection" (Tapia et al., 2016). Another study conducted between October 2014 and June 2015 tested the safety and immunogenicity of ChAd3 on 120 participants in Switzerland (De Santis et al., 2016). They also concluded that the vaccine was safe, although there were mild to moderate adverse effects, including fatigue and headache. The vaccine was shown to be immunogenic even after 6 months and the investigators recommended its use in phase 2 and 3 trials.

Another study conducted between February and April 2015 tested the safety and immunogenicity of ChAd3 and rVSV on 1500 participants in Liberia (Kennedy et al., 2017). One month after treatment, the vaccine had elicited an immune response which was sustained over 12 months and no safety concerns were identified (Kennedy et al., 2017). Finally, another study was conducted beginning March 2015 to determine the effectiveness of ZMapp in a total 72 patients in Liberia, Sierra Leone, Guinea, and the United States. Patients received either ZMapp and the standard of care or the standard of care alone. In the control group, 37\% of people died compared to $22 \%$ in the experimental group, which meant that Zmapp was $91.2 \%$ superior to the standard of care alone, although it did not meet the $97.5 \%$ threshold to qualify as effective (The PREVAIL II Writing Group, 2016). All five studies had one thing in common: they showed that the treatments were safe and potentially effective. The principle of equipoise requires balancing the risks and benefits of experimental treatments compared to conventional care (Adebamowo et al. 2014). As mentioned earlier, the mortality rate was as high as $70 \%$ in some regions following conventional care measures. Hence, the high mortality rate with conventional care illustrates that this epidemic was time-sensitive, as it was rapidly killing patients. The results of the study suggest that because of the need to act quickly, the risk was worth taking as the experimental treatments were unlikely to be riskier than the conventional care. The principle of equipoise would therefore dictate that the drugs be made available to all patients. One can thus argue that they should have been distributed in non-randomized studies to potentially reduce the number of deaths.

Another reason why RCTs were not the best approach is the fact that they were so controversial and led to long debates which delayed intervention. While the epidemic was declared in March 2014, the first RCT began in September 2014 as a result of the debate over the best design to use (Ewer et al., 2016). This delay weakened the trial because there were few available patients for enrollment after it formally started. Two studies addressed this issue. The RCT conducted in Liberia was initially planned to be both phase 3 (which would involve Ebola patients to determine the efficacy of the experimental therapies) and phase 2 trials (to determine safety, by using healthy subjects) (Kennedy et al., 2017). However, because of the decline in new Ebola cases, investigators were unable to enroll enough Ebola patients, and could only enroll healthy patients and assess safety and immunogenicity in a phase 2 trial (Kennedy et al., 2017). The study was conducted in 2015, and investigators were unable to test the vaccines on enough patients to determine its effectiveness. Similarly, the ZMapp study described above also needed to start the experiments early since it had been proven to be effective in non-human primates in the 5-day window after infection (The PREVAIL II Writing Group, 2016). In reality, it was difficult to find and recruit patients in the midst of the epidemic, and the patients ultimately enrolled were more than one week past their date of infection. 
For those people enrolled in the ZMapp trial, those who died in the experimental group died before receiving their second of three programmed ZMapp injections (The PREVAIL II Writing Group, 2016). Patients already had the disease for quite some time before enrolling in the trial, which implies that the trial did not test the full potential of this drug. The fact that they did not even receive two of the programmed injections and ZMapp was still 91.5\% superior to the standard of care shows that it could be more effective. Finally, the authors attribute their small sample size to the decline in cases (The PREVAIL II Writing Group, 2016). A larger sample size may have highlighted the effectiveness of ZMapp to a greater extent. This again shows that the delay in starting the intervention were due to the debates regarding the most appropriate study design, leading to a small sample size for the trials and thus an ineffective assessment of the treatments. While this may suggest that RCTs would have been beneficial if they had started early, it actually highlights why they were problematic: the controversy that arose over their use delayed intervention, which was detrimental because the ability of researchers to generate valid data regarding the treatments required an earlier start.

Finally, RCTs were not the best approach to address the epidemic because the typical process of conducting drug trials ensured that patients would not have acquired the treatments in a timely manner. Even if the trials had started on time, it might have still taken too long before the treatments were offered to patients. This is first due to the need to conduct safety trials. Four of the studies described earlier were safety and immunogenicity trials. Because Phase 1 trials are required before conducting phase 2 or phase 3 trials, this would have further delayed the date of treatment. Additionally, scientific data must be repeatable. The safety trials for the ChAd3 vaccine were conducted in the US, the UK, Switzerland and Mali, and they all confirmed that the vaccine was safe, which was necessary to make sure that the data is reliable. Even if the trials had started earlier, conducting all these safety trials in different locations would have also delayed the intervention. It would have been more efficient to conduct the safety trials in the areas affected first, and move on to efficacy trials. Finally, the trials themselves lasted between 2 and 12 months. This is relatively long considering that Ebola patients were dying rapidly. Because it would have taken so long to generate significant data, the principle of equipoise again requires that the treatments be made widely available. The principle of equipoise insists that as long as there is uncertainty over the risks and benefits of the experimental treatments, safety trials must be conducted and valid data must be generated through randomization. However, because of the need to act quickly and the high mortality rates, the time it would have taken to generate data in order to help patients provides additional evidence for the argument that RCTs were not the best approach.

\section{CONCLUSION:}

As this paper has shown, there were valid arguments on both sides of the debate for the appropriateness of RCTs during the 2014-2015 Ebola epidemic. On one hand, proponents of RCTs focused on the need to generate robust data, the safety of patients, and the benefit to society as a whole. On the other hand, opponents of RCTs focused on high mortality rate, the need to preserve patient-doctor trust, and the benefit to individual patients. One interesting dilemma that arose 
from these arguments is whether the priority of researchers lies in observing small improvements or more obvious ones through the use of experimental treatments. Some proponents of RCTs argued that this type of experimental design detects small differences between the two groups, but opponents argued that RCTs are not necessary because as long as the drug is significantly effective, removing all the confounding variables is not required.

A close look at five RCTs conducted during and after the outbreak led to the conclusion that RCTs were not the best approach because the principle of equipoise required that treatments be made available to patients. Alternatively, RCTs should have been started earlier to ensure a high enough sample size for the data to be valid. All of the studies found that the experimental treatments were safe and immunogenic. Thus, the treatments should have been distributed to patients because the studies showed that the patients would not have been in greater risk than with conventional care. Additionally, the delay in interventions, due to the debates over the appropriateness of RCTs, made it more difficult for researchers to gather data because cases began to decline. Finally, the actual process of conducting clinical trials was another roadblock because it ensured that the patients would not be provided with the treatments on time. Ultimately, the findings from these and subsequent RCTs add to the knowledge on treatments for EVD and will be beneficial to future patients. The next epidemic will be less challenging because there will be some knowledge on the safety and potential efficacy of these treatments, which means that healthcare workers will be better armed. This fact is not surprising because, as mentioned above, the goal of RCTs is not to help individual patients but to generate knowledge that will be beneficial to society as a whole, and especially future generations.

\section{ACKNOWLEDGMENTS}

I would like to thank Professor Melissa Graboyes for her assistance throughout the writing of this research paper. Her expertise on this topic, her advices on the process of refining my topic and writing definitely contributed to my success in completing this research project. Professor Graboyes was willing to read my paper a few times to give me constructive criticism. I'm very thankful to her. I would also like to thank the Honor's College (and all the professors who have taught my courses) for helping me improve my writing and research skills. Finally, I would like give special thanks to my family and friends for the constant encouragement and the prayers, as these help me persevere and work hard. I'm especially thankful to my parents for their all of their sacrifices which enabled me to come to this country and have more opportunities. Thank you all for your contribution to this project!

\section{REFERENCES}

Adebamowo, C., Bah-Sow, O., Binka, F., Bruzzone, R., Caplan, A., Delfraissy, J.-F., ... Whitehead, J. (2014). Randomised controlled trials for Ebola: practical and ethical issues. The Lancet, 384(9952), 1423-1424. https://doi.org/10.1016/So140-6736(14)61734-7

Calain, P. (2018). The Ebola clinical trials: a precedent for research ethics in disasters. Journal of Medical Ethics, 44(1), 3-8. https://doi.org/10.1136/medethics-2016-103474 
Check Hayden, E. (2014). Ethical dilemma for Ebola drug trials. Nature, 515(7526), 177-178. https://doi.org/10.1038/515177a

Cohen, J., \& Kupferschmidt, K. (2014). Ebola vaccine trials raise ethical issues. Science, 346(6207), 289-290. https://doi.org/10.1126/science.346.6207.289

Coltart, C. E. M., Lindsey, B., Ghinai, I., Johnson, A. M., \& Heymann, D. L. (2017). The Ebola outbreak, 2013-2016: old lessons for new epidemics. Philosophical Transactions of the Royal Society B: Biological Sciences, 372(1721), 20160297. https://doi.org/10.1098/rstb.2016.0297

Cox, E., Borio, L., \& Temple, R. (2014). Evaluating Ebola Therapies - The Case for RCTs. New England Journal of Medicine, 371(25), 2350-2351. https://doi.org/10.1056/NEJMp1414145

De Santis, O., Audran, R., Pothin, E., Warpelin-Decrausaz, L., Vallotton, L., Wuerzner, G., ... Genton, B. (2016). Safety and immunogenicity of a chimpanzee adenovirus-vectored Ebola vaccine in healthy adults: a randomised, double-blind, placebo-controlled, dose-finding, phase 1/2a study. The Lancet Infectious Diseases, 16(3), 311-320.

https://doi.org/10.1016/S1473-3099(15)00486-7

Ewer, K., Rampling, T., Venkatraman, N., Bowyer, G., Wright, D., Lambe, T., ... Hill, A. V. S. (2016). A Monovalent Chimpanzee Adenovirus Ebola Vaccine Boosted with MVA. New England Journal of Medicine, 374(17), 1635-1646.

https://doi.org/10.1056/NEJMoa1411627

Kanapathipillai, R., Henao Restrepo, A. M., Fast, P., Wood, D., Dye, C., Kieny, M.-P., \& Moorthy, V. (2014). Ebola Vaccine - An Urgent International Priority. New England Journal of Medicine, 371(24), 2249-2251. https://doi.org/10.1056/NEJMp1412166

Kennedy, S. B., Bolay, F., Kieh, M., Grandits, G., Badio, M., Ballou, R., ... Lane, H. C. (2017). Phase 2 Placebo-Controlled Trial of Two Vaccines to Prevent Ebola in Liberia. New England Journal of Medicine, 377(15), 1438-1447. https://doi.org/10.1056/NEJMoa1614067

Lanini, S., Zumla, A., Ioannidis, J. P. A., Caro, A. D., Krishna, S., Gostin, L., ... Ippolito, G. (2015). Are adaptive randomised trials or non-randomised studies the best way to address the Ebola outbreak in west Africa? The Lancet Infectious Diseases, 15(6), 738-745. https://doi.org/10.1016/S1473-3099(15)70106-4

Tapia, M. D., Sow, S. O., Lyke, K. E., Haidara, F. C., Diallo, F., Doumbia, M., ... \& Sztein, M. B. (2016). Use of ChAd3-EBO-Z Ebola virus vaccine in Malian and US adults, and boosting of Malian adults with MVA-BN-Filo: a phase 1, single-blind, randomised trial, a phase 1b, open-label and double-blind, dose-escalation trial, and a nested, randomised, double-blind, placebo-controlled trial. The Lancet infectious diseases, 16(1), 31-42. 
The PREVAIL II Writing Group. (2016). A Randomized, Controlled Trial of ZMapp for Ebola Virus Infection. New England Journal of Medicine, 375(15), 1448-1456. https://doi.org/10.1056/NEJMoa1604330

Upshur, R., \& Fuller, J. (2016). Randomized controlled trials in the West African Ebola virus outbreak. Clinical Trials: Journal of the Society for Clinical Trials, 13(1), 10-12. https://doi.org/10.1177/1740774515617754

World Health Organization. (2018, February 12). Ebola virus disease. Retrieved from www.who.int/news-room/fact-sheets/detail/ebola-virus-disease.

World Health Organization. (2014, August 12). Ethical Considerations for Use of Unregistered Interventions for Ebola Virus Disease (EVD). Retrieved from www.who.int/mediacentre/news/statements/2014/ebola-ethical-review-summary/en/. 